\title{
INTERCHANGING ITERATED INTEGRATION
}

\section{LAWRENCE LESSNER}

\begin{abstract}
If $k(x, y)$ is a measurable, real valued, finite a.e. function on $X \times Y$, then necessary and sufficient conditions are given for the two iterated Lebesgue integrals of $k(x, y)$ to be equal and finite by employing Saks' theorem on the convergence of a sequence of finite measures and the Vitali convergence theorem. The conditions, more general than those of either Fubini's or Tonelli's theorems in this case, are applied to an example of a nonintegrable function to show that its iterated integrals are in fact equal and finite.
\end{abstract}

Introduction. In every instance when either Fubini's or Tonelli's theorem is applied to show that

$$
\iint k(x, y) d y d x=\iint k(x, y) d x d y<\infty
$$

we are also able to assert

$$
\int \chi(E) \int \chi(F) k(x, y) d y d x=\int \chi(F) \int \chi(E) k(x, y) d x d y<\infty
$$

for all $F \in \mathbb{Q}_{1}$ and $E \in \mathbb{Q}_{2}$. In this paper we give necessary and sufficient conditions for the interchange of integration in (*) by applying Saks' theorem on the convergence of a sequence of finite measures and the Vitali convergence theorem.

Defintions and Notation. Let $\left(Y, \mathbb{Q}_{1}, \mu_{1}\right)$ and $\left(X, \mathbb{Q}_{2}, \mu_{2}\right)$ be $\sigma$-finite measure spaces, and $\mu_{1}$ and $\mu_{2}$ nonnegative measures defined on $Q_{1}$ and $\mathbb{Q}_{2}$. Let $Q_{2} \times Q_{1}$ be the completed product measure defined on $X \times Y, \chi(E)$ will denote the characteristic function of the set $E$, and $\int k(x, y) d y$ will mean the usual Lebesgue integral defined by

$$
\mu_{1}: \int \chi(Y) k(x, y) d \mu_{1}(y) \text {. }
$$

Similarly $\int k(x, y) d x=\int \chi(X) k(x, y) d \mu_{2}(x)$. We shall assume all functions are real valued a.e. and that $\delta<\infty$ will mean that $\delta$ is a finite real number.

THEOREM 1. Let $k(x, y)$ be any $\mathbb{Q}_{2} \times \mathbb{Q}_{1}$ measurable function on $X_{2} \times X_{1}$, then for all $E \in \mathbb{Q}_{2}, F \in \mathbb{Q}_{1}$ we have

Presented to the Society, January 28, 1977; received by the editors August 15, 1975.

AMS (MOS) subject classifications (1970). Primary 28A25, 28A40, 46G99, 47A05, 47B99.

Key words and phrases. Iterated integrals, Saks' theorem, Fubini-Tonelli's theorem, and Vitali convergence theorem. 


$$
\int \chi(E) \int \chi(F) k(x, y) d y d x=\int \chi(F) \int \chi(E) k(x, y) d x d y<\infty
$$

if and only if for all $F \in \mathbb{Q}_{1}, \iint \chi(F) k(x, y) d y d x<\infty$ and $\int k(x, y) d x<\infty$, $\mu_{1}$ a.e.

Proof OF THEOREM. The conditions stated in the theorem are clearly necessary for the change in the order of integration of the iterated integral. We shall proceed with the proof of sufficiency.

Let $\left\{x_{j}\right\}_{j}$ be any countable collection of disjoint $\mathbb{Q}_{2}$ measurable sets of finite $\mu_{2}$ measure where $\cup_{j} x_{j}=X_{2}$. Since $\iint k(x, y) d y d x<\infty$, we have $\left|\int k(x, y) d y\right|<\infty, \mu_{2}$ a.e. Consequently, $\int|k(x, y)| d y<\infty, \mu_{2}$ a.e.

Let $\Omega(n)=\left\{x \in \cup_{1}^{n} x_{j}: \int|k(x, y)| d y \leqslant n\right\}$, then $\Omega(n) \subset \Omega(n+1)$ and $\cup_{1}^{\infty} \Omega(n)=X$ a.e. Also

$$
0 \leqslant \int \chi(\Omega(n)) \int|k(x, y)| d y d x \leqslant \int \chi\left(\bigcup_{1}^{n} x_{j}\right) \cdot n d x<\infty .
$$

So by the usual Fubini-Tonelli theorem, we have for all $E \in \mathbb{Q}_{2}$ and $F \in \mathbb{Q}_{1}$,

$$
\begin{aligned}
\int \chi(E \cap \Omega(n)) \int \chi(F) k(x, y) d y d x & \\
& =\int \chi(F) \int \chi(E \cap \Omega(n)) k(x, y) d x d y<\infty .
\end{aligned}
$$

Define, for $E \in \mathbb{Q}_{2}$ fixed, $\mu_{n}(F)=\int \chi(E \cap \Omega(n)) \int \chi(F) k(x, y) d y d x$; then since

$$
\mu_{n}(F)=\int \chi(F) \int \chi(E \cap \Omega(n)) k(x, y) d x d y,
$$

we know that $\mu_{n}$ is a finite measure on $\mathbb{Q}_{1}$ and $\mu_{n} \ll \mu_{1}$.

Now $f_{n}=\chi(E \cap \Omega(n)) \int \chi(F) k(x, y) d y$ is $\mu_{2}$ integrable, $f_{n}$ converges to $\chi(E) \int \chi(F) k(x, y) d y, \quad \mu_{2}$ a.e., where $\left|f_{n}\right| \leqslant\left|\int \chi(F) k(x, y) d y\right|$ and $\int \chi(F) k(x, y) d y$ is an integrable function by hypothesis. Consequently an application of the Lebesgue dominated convergence theorem implies that for each $F \in \mathbb{Q}_{1}$, the $\operatorname{limit}_{n \rightarrow \infty} \mu_{n}(F)$ exists and has a finite value

$$
\mu(F)=\int \chi(E) \int \chi(F) k(x, y) d y d x .
$$

By Sak's theorem, see [1, p. 332] or [3, p. 158], we know that the functions

$$
g_{n}(y)=\int \chi(E \cap \Omega(n)) k(x, y) d x
$$

satisfy the following two conditions:

(1) Uniform absolute continuity; for all $0<\varepsilon$, there exists $0<\delta$ such that if $\mu_{1}(F)<\delta$, then $\sup _{n} \int \chi(F)\left|g_{n}(y)\right| d y<\varepsilon$.

(2) Equicontinuity at zero from above; for any decreasing sequence $\left\langle F_{j}\right\rangle$ of $Q_{1}$ measurable sets where $\bigcap_{j} F_{j}$ is $\mu_{1}$ null we have

$$
\lim _{j \rightarrow \infty} \sup _{n} \int \chi\left(F_{j}\right)\left|g_{n}(y)\right| d y=0 .
$$


An application of Lebesgue's dominated convergence theorem shows that $g_{n}(y)$ converges $\mu_{1}$ a.e. to $g(y)=\int \chi(E) k(x, y) d x$. By a version of the Vitali convergence theorem, see [2, p. 108], we see that $\lim _{n \rightarrow \infty} \int\left|g_{n}-g\right| d y=0$. Consequently

$$
\begin{aligned}
\int \chi(E) \int \chi & (F) k(x, y) d y d x \\
& =\lim _{n \rightarrow \infty} \mu_{n}(F)=\lim _{n \rightarrow \infty} \int \chi(F) g_{n}(y) d y \\
& =\int \chi(F) \int \chi(E) k(x, y) d x d y<\infty .
\end{aligned}
$$

The above proof is also a demonstration of the following result. If for all $F \in Q_{1}$ we have $\iint k(x, y) \chi(F) d y d x<\infty$ and $\int k(x, y) d x<\infty, \mu_{1}$ a.e., then $v(F)=\iint \chi(F)(y) k(x, y) d y d x$ is a countably additive, finite signed measure of bounded variation which is absolutely continuous with respect to $\mu_{1}$ and has Radon-Nikodým derivative $d v / d \mu_{1}=\int k(x, y) d x$; see [3, p. 128].

EXAMPLE. We conclude this paper with an application of Theorem 1. The so called discrete version of the Hilbert transform, discussed in [4, p. 170], may be summarized as follows. Let $N=\{2,3,4, \ldots\}$ be equipped with the counting measure and define $t(i, j): N \times N \rightarrow(-\infty, \infty)$ by $t(i, j)=(i-$ $j)^{-1}$ for $i \neq j$ and $t(i, j)=0$ for $i=j$, then $t(i, j)$ defines an integral operator $H: l_{2} \rightarrow l_{2}$, where $l_{2}$ is the class of square integrable functions on $N$. Thus for $f \in l_{2}, f$ is the square summable sequence $\langle f(j)\rangle_{j}$ and $\int t(i, j) f(j) d_{j}=$ $\sum_{j=1}^{\infty} t(i, j) f(j) \in l_{2}$. In [4, p. 170], it is shown that

$$
\sum_{i=1}^{\infty} \sum_{j=1}^{\infty}(\sqrt{i j}|i-j| \ln i \ln j)^{-1}=+\infty .
$$

Also since $f=\left\langle(\sqrt{j} \ln j)^{-1}\right\rangle_{j} \in l_{2}$ we have $f(i) H(f)(i)=g(i) \in l_{1}$ by Hölder's inequality. If we define for $h \in l_{\infty}, S(h)=f H(f h)$, then $S: l_{\infty} \rightarrow l_{1}$ and $S$ is in fact an integral operator with kernel

$$
s(i, j)=((i-j) \sqrt{i j} \ln i \ln j)^{-1}
$$

for $i \neq j$ and equal to zero otherwise.

Now let $\chi(n)=\chi([n-1 / 2, n+1 / 2))$ and define $k(x, y):[1.5, \infty) \times$ $[1.5, \infty) \rightarrow(-\infty, \infty)$ by

$$
k(x, y)=\sum_{i=1}^{\infty} \sum_{j=1}^{\infty} s(i, j) \chi(i)(x) \chi(j)(y) .
$$

Clearly $k(x, y)$ is well defined since for a given $(x, y)$, at most one of the collection $\{x(i)(x) \chi(j)(y)\}_{i j}$ is not zero. If we take $[1.5, \infty)$ with the usual Lebesgue measure, $\lambda$, for the real line, then we shall show that for every measurable set $E \subset[1.5, \infty), \int \chi(E)(y) k(x, y) d y d x<\infty$.

Well 


$$
\begin{aligned}
\int|k(x, y)| d y & =\sum_{i} \chi(i)(x) \int \sum_{j}|s(i, j)| \chi(j)(y) d y \\
& =\sum_{i}\left(\sum_{j}|s(i, j)|\right) \chi(i)(x)<\infty, \quad \lambda \text { a.e. }
\end{aligned}
$$

since

$$
\begin{aligned}
\sum_{j}|s(i, j)| & =(\sqrt{i} \ln i)^{-1}\left[\sum_{j=2}^{\infty}(\sqrt{j}|i-j| \ln j)^{-1}\right] \\
= & (\sqrt{i} \ln i)^{-1}\left[\sum_{j=2}^{i}(\sqrt{j}|i-j| \ln j)^{-1}+\sum_{p=1}^{\infty}(p \sqrt{p+i} \ln (p+i))^{-1}\right]
\end{aligned}
$$

and $\sum_{p=1}^{\infty}(p \sqrt{p+i} \ln (p+i))^{-1}$ converges by comparison with $\sum_{p=1}^{\infty} p^{-3 / 2}$. Thus $\int \chi(E)(y) k(x, y) d y<\infty$ a.e. and

$$
\begin{aligned}
\int \chi(E)(y) k(x, y) d y & =\sum_{i} \chi(i)(x) \int \chi(E)\left(\sum_{j} s(i, j) \chi(j)(y)\right) d y \\
& =\sum_{i}\left(\sum_{j} s(1, j) \int \chi(E) \chi(j)(y)\right) \chi(i)(x) \\
& =\sum_{i}\left(\sum_{j} s(i, j) \lambda(j)\right) \chi(i)(x)
\end{aligned}
$$

where $\lambda(j)=\lambda(E \cap[j-1 / 2, j+1 / 2))$. Thus

$$
\begin{aligned}
\left|\iint \chi(E) k(x, y) d y d x\right| & =\left|\int \sum_{i}\left(\sum_{j} s(i, j) \lambda(j)\right) \chi(i)(x) d x\right| \\
& \leqslant \sum_{i}\left|\sum_{j} s(i, j) \lambda(j)\right|=\sum_{i}|S(\phi)|<\infty
\end{aligned}
$$

because $\phi=\langle\lambda(j)\rangle \in l_{\infty}, S: l_{\infty} \rightarrow l_{1}$, and $\|S(\phi)\|_{1}=\Sigma_{i}|S(\phi)(i)|$.

Since $k(x, y)=k(y, x)$ and $\int|k(x, y)| d y<\infty$, we must have $\int|k(x, y)| d x$ $<\infty$ a.e. Thus we may apply Theorem 1 to show that for any measurable sets $E, F \subset[1.5, \infty)$,

$$
\int \chi(E) \int \chi(F) k(x, y) d x d y=\int \chi(F) \int \chi(E) k(x, y) d y d x<\infty
$$

even though

$$
\iint|k(x, y)| d y d x=\sum_{i} \sum_{j}|s(i, j)|=+\infty
$$




\section{BIBLIOGRAPHY}

1. A. C. Zaanen, Integration, North-Holland Publishing Company, Amsterdam, 1967.

2. P. R. Halmos, Measure theory, D. Van Nostrand Company, New York, 1950.

3. N. Dunford and J. T. Schwartz, Linear operators, Part I, Interscience, New York, 1967.

4. A. L. Peressini, Ordered topological vector spaces, Harper and Row, New York, 1967.

Department of Mathematics, Northrop University, Inglewood, Callfornia 90306 